\title{
Regulation of bacterial Type III Secretion System export gate opening
}

\section{Authors}

Owain J. Bryanta, ${ }^{a,{ }^{*},}$, Gillian M. Fraser ${ }^{a,{ }^{*}}$

\section{Affiliations}

aDepartment of Pathology, University of Cambridge, Tennis Court Road, Cambridge, CB2 1QP, United Kingdom

\section{Corresponding authors}

*Gillian M. Fraser

Department of Pathology, University of Cambridge, Tennis Court Road, Cambridge, CB2 1QP, United Kingdom

Phone: +44 1223330245 Email: gmf29@cam.ac.uk

*Owain J. Bryant

Department of Pathology, University of Cambridge, Tennis Court Road, Cambridge, CB2 1QP, United Kingdom

Phone: +441223 366145 Email: owain.bryant@bioch.ox.ac.uk

${ }^{\mathrm{b} C u r r e n t}$ address: Department of Biochemistry, University of Oxford, South Parks Road, Oxford, OX1 3QU

\section{Keywords}

Type III secretion; protein export; bacterial flagella biogenesis 


\section{Abstract}

2 Type III Secretion Systems (T3SS) transport proteins from the bacterial cytosol for

3 assembly into cell surface nanomachines or for direct delivery into target eukaryotic

4 cells. At the core of the flagellar T3SS, the FlhAB-FliPQR export gate regulates

5 protein entry into the export channel whilst maintaining the integrity of the cell membrane. Here, we identify critical residues in the export gate FliR plug that stabilise the closed conformation, preserving the membrane permeability barrier, and we show that the gate opens and closes in response to export substrate availability.

9 Our data indicate that FlhAB-FliPQR gate opening, which is triggered by substrate export signals, is energised by FlhA in a proton motive force-dependent manner. We

11 present evidence that the export substrate and the FliJ stalk of the flagellar ATPase

12 provide mechanistically distinct, non-redundant gate-activating signals that are

13 critical for efficient export.

\section{Introduction}

16 Type III Secretion Systems (T3SS) transport proteins across the bacterial inner and

17 outer membranes and, in the case of the virulence T3SS (vT3SS), across the

18 plasma membrane of target eukaryotic cells ${ }^{1},{ }^{2,},{ }^{3}$. The flagellar T3SS (fT3SS) is

19 required for assembly of rotary flagella, which facilitate cell motility ${ }^{1}, 2,3,4$. Despite their

different functions, the vT3SS and fT3SS are evolutionarily related and contain conserved core export components $5,6,7,8,9,10,11$.

For construction of the bacterial flagellum, most of the flagellar components

23 are exported by the fT3SS machinery, which is embedded in the cell envelope. The core of the export machinery is made up of five highly conserved proteins (FlhA,

25 FlhB, FliP, FliQ and FliR). FlhA forms a nonameric ring structure comprising a 
cytoplasmic domain which functions as a docking platform for export cargo and an

27 N-terminal region (FlhAN) with putative proton conducting activity $5,12,13,14$. Recent

28 cryo-ET data has shown that FlhAN wraps around the base of a helical-dome

composed of FliPQR and the N-terminal region of FlhB $\left(\mathrm{FlhB}_{\mathrm{N}}\right)^{13}$. Together, FlhA and

$P Q R B_{N}$ constitute the ABPQR export gate, which regulates entry of flagellar subunits into the central channel that runs the length of the nascent flagellum $5,12,13$. In addition to the ABPQR export gate, an ATPase complex (FliHIJ) located in the cytoplasm converts the fT3SS into a highly efficient pmf-driven protein export machine ${ }^{10,15,16,17}$. Recent structural studies of FliPQR and $\mathrm{FlhB}_{\mathrm{N}}$ have shown that multiple interactions stabilise the closed export gate ${ }^{5,6},{ }^{12},{ }^{18}$. A central plug formed by residues $106-120$ of $\mathrm{FliR}$, a methionine rich loop (M-gate) within FliP, and ionic interactions between adjacent FliQ subunits maintain the closed conformation ${ }^{5}, 6,18$.

38 Structural analysis of the export gate in the open conformation revealed multiple rearrangements within the M-gate and an upward displacement of the FliR plug, indicating that energy must be provided to open the gate and permit subunit

41 translocation across the inner membrane ${ }^{18}$. We reasoned that, as the PQRB components of the gate are positioned adjacent to the proposed proton conducting FlhA membrane protein, FlhA might use the energy derived from the pmf to promote gate opening. Moreover, we hypothesised that gate opening must be regulated by one or more factors to permit subunit export whilst maintaining the membrane permeability barrier. We set out to test these hypotheses experimentally. 
51 Replacement of critical residues in the FliR plug destabilises the closed

52 conformation of the fT3SS export gate.

53 The fT3SS ABPQR export gate resides in a closed conformation in the absence of

54 subunit export ${ }^{5},{ }^{6},{ }^{13},{ }^{11},{ }^{18}$. We set out to engineer the export gate to destabilise the

55 closed conformation and favour the open state, reasoning that mutation of contact

56 points between adjacent gate subunits would reduce the energy barrier required for

57 gate opening (Fig.1A). To screen for potential gate opening mutants, we used a subunit variant $\left(F l g D_{\text {short }}\right)$ that we have previously shown is unable to trigger efficient opening of the export gate ${ }^{19}$. We predicted that mutations which promoted export

60 gate opening would also supress the FlgD short motility defect. Recent structural information has revealed the potential gating mechanism of the FliPQR gate components. A region of FliR (residues 106-121) forms a plug that occludes the gate channel, and this plug must be displaced to achieve gate opening ${ }^{18}$. In the FliR plug, residues Phe-110 and Phe-113 make multiple contacts within the core of the export gate, and might have a central role in gate opening ${ }^{5},{ }^{12}$. To assess the importance of selected FliR residues in gate opening, we replaced Phe-110 and/or Phe-113 with alanine, and replaced Gly-117 with a bulkier charged aspartate residue and screened for suppression of the FlgD short motility defect (Fig. 1). We found that all three of these FliR-plug mutations $\left(F_{110} A, F_{113} A\right.$ and $\left.G_{117} D\right)$ supressed the $F_{1 g} D_{\text {short }}$

70 motility defect, yet did not increase export or motility of cells encoding wild type FlgD

71 (Fig. 1B, 1C and S1, S2), indicating these mutations destabilise the closed export gate conformation. The FliR-G ${ }_{117} \mathrm{D}$ mutation was previously proposed to rescue an interaction between FliR and FlhA, but the data presented here indicate that the 
$74 \mathrm{G}_{117} \mathrm{D}, \mathrm{F}_{113} \mathrm{~A}$ and $\mathrm{F}_{115} \mathrm{~A}$ mutations destabilise the closed conformation of the export gate (Fig. 1D) 20

We hypothesised that deletion of the FliR plug would promote the open gate

conformation and, as a result, increase the permeability of the inner membrane.

79 Bacterial cells that fail to maintain the permeability barrier across the inner

membrane are more sensitive to a number of chemical agents, such as choline ${ }^{21}$. To

determine whether a strain encoding a FliR plug deletion variant (FliR $\Delta 110-116$,

hereafter termed FliR $\Delta$ plug) was sensitive to choline, we performed growth inhibition assays by culturing cells in media containing increasing concentrations of choline (Fig 1E). We found that the FliR $\Delta$ plug strain was sensitised to choline compared to wild type Salmonella, indicating that the FliR plug is important for formation of a tight barrier that is impermeable to small molecules, and for maintaining the closed conformation of the fT3SS export gate (Fig. 1E, 1F, S3).

The export gate fluctuates between open and closed states in response to substrate availability

A previous study showed the $\mathrm{M}_{210} \mathrm{~A}$ mutation in the FliP component of the export gate sensitised cells to a variety of chemical agents ${ }^{21}$. This sensitivity could be reversed by trapping a subunit in the export channel, suggesting that the export gate can form an effective seal around a substrate during transit ${ }^{21}$. We reasoned that if the export gate fluctuates between open and closed conformations in response to the availability of substrate, strains producing fewer flagellar subunits would, at any given time, have fewer subunits transiting through the fT3SS to seal the leaky 
98 FliR $\Delta$ plug gate. To test this, we constructed a strain deleted for the genes that

99 encode most of the early flagellar subunits by replacing a large portion of the flg

100 operon ( $f \lg B, f l g C, f l g D, f l g E, f l g F, f l g G, f l g H, f l g l$ and $f l g J)$ with a kanamycin

101 resistance cassette. In addition, we constructed a second strain in which the FliR

102 plug and the rod/hook genes were deleted. This FliR $\Delta$ plug- $\Delta$ subunit strain was

103 significantly more sensitive to choline than the FliR $\Delta$ plug strain, indicating that

104 actively transiting subunits partially seal the export gate when the FliR plug is absent

105 (Fig. 1E, 1F, S3). Notably, the $\Delta$ subunit strain (which produces wild type FliR) was

106 not more sensitive to choline than wild type Salmonella, indicating that decreased

107 subunit availability does not increase the permeability of the wild type export gate to

108 small molecules (Fig. 1E, 1F, 1G). This suggests that the export gate must fluctuate

109 between open and tightly closed conformations, in response to availability of

110 subunits at the export machinery.

111

112 Destabilising the closed conformation of the export gate alleviates the export

113 defect associated with a variant of the gate component FIhA.

114 Opening of the export gate is thought to be energised, in part, by harnessing of the

115 pmf by FlhA. A previous study proposed that FlhA interacts with FliR, as mutations in

116 fliR were found to suppress the motility defect of a weakly motile Salmonella $(\Delta$ fliH-

$117 \mathrm{flil}$, flhB-P $\mathrm{P}_{28} \mathrm{~T}, \mathrm{flh} A-\mathrm{K}_{203} \mathrm{~W}$ ) that contained deletions in flagellar ATPase genes (flil and

$118 \mathrm{fliH}$ ), a suppressor mutation in $\mathrm{flhB}, \mathrm{P}_{28} \mathrm{~T}$, which overcomes the loss of FliH and Flil,

119 and a mutation in $f / h A, \mathrm{~K}_{203} \mathrm{~W}$, that attenuates motility and export ${ }^{20}$. However,

120 structural studies subsequently revealed that these FliR suppressor mutations are

121 located in the core of the FliPQR complex, indicating that this region of FliR is unable 
122 to contact FlhA within the assembled fT3SS 5 . We have shown that one of these

123 suppressor mutations in the FliR plug (FliR-G $\left.{ }_{117} \mathrm{D}\right)$ destabilises the closed

124 conformation of the export gate (Fig. 1B and 1C). Based on these data, we

125 hypothesised that FliR-G ${ }_{117} \mathrm{D}$ might suppress the motility and export defects

126 associated with mutation of FlhA- $\mathrm{K}_{203}$ by destabilising the closed conformation of the

127 export gate, rather than by restoring an interaction between FliR and FlhA. To test

128 this, we assessed whether the export and motility of strains encoding FlhA-K $\mathrm{K}_{203} \mathrm{~A}$

129 could be recovered by introducing mutations (FliR-F $F_{113} A$ or FliR- $\left.G_{117} D\right)$ that

130 destabilise the closed gate conformation. We found that, as predicted, FliR- $\mathrm{F}_{113} \mathrm{~A}$

131 and FliR-G ${ }_{117} \mathrm{D}$ suppressed the export and motility defects associated with FlhA

$132 \mathrm{~K}_{203} \mathrm{~A}$ (Fig. 2). The data indicate that the $f / h A-\mathrm{K}_{203} \mathrm{~A}$ strain is unable to open the

133 export gate efficiently and that introduction of fliR mutations that destabilise the

134 closed gate might lower the energy barrier that must be overcome by FlhA-K ${ }_{203} \mathrm{~A}$ to 135 open the gate (Fig. 2A-2B).

137 Increasing the pmf suppresses the FIhA-K ${ }_{203}$ A export defect

138 Our results support the view that FlhA has a role in opening the export gate and that

139 FlhA-K $\mathrm{K}_{203}$ is critical for efficient gate opening. As FlhA has been proposed to facilitate

140 subunit export by functioning as a proton-conducting channel ${ }^{14}, 17,20$, we

141 hypothesised that the FlhA- $\mathrm{K}_{203} \mathrm{~A}$ mutation might render the export machinery unable

142 to couple pmf to efficient gate opening. If this were the case, the gate opening and

143 subunit export defects of a strain carrying chromosomally encoded $f / h A-K_{203} A$ might

144 be overcome by increasing the pmf. To test this, we artificially increased the pmf

145 across the bacterial inner membrane by driving intracellular proton consumption 22 . 
146 Cells supplemented with arginine convert intracellular protons and arginine to

147 agmatine and $\mathrm{CO}_{2}$ through the action of the cytoplasmic enzyme arginine

148 decarboxylase, which effectively acts as a proton sink in the cytosol and therefore

149 increases the $\mathrm{pmf}^{22}$ (Fig. 3A). We performed a modified subunit export assay in

150 which cells were grown to mid-exponential phase, washed, resuspended in media containing $20 \mathrm{mM}$ arginine and culture supernatants were collected after 30 mins.

Addition of arginine to cultures of the $f / h A-\mathrm{K}_{203} \mathrm{~A}$ strain increased subunit export by $40 \%$, whereas subunit export in the wild type strain was unchanged (Fig. 3B).

154 Deletion of the two arginine decarboxylase enzymes (spe $A$ and adi $A$ ) encoded by

Salmonella abolished the arginine-dependent increase in subunit export by the flhA-

$156 \mathrm{~K}_{203} \mathrm{~A}$ strain, indicating that the $\mathrm{FlhA}-\mathrm{K}_{203} \mathrm{~A}$ mutation prevented efficient use of the

157 pmf by the export machinery. The data are consistent with wild type FlhA utilising the 158 pmf to energise export gate opening (Fig. S4).

Two distinct non-redundant signals activate the pmf-driven export machinery

161 We have found that the $\mathrm{N}$-terminus of rod/hook subunits contains a hydrophobic signal that triggers opening of the export gate ${ }^{19}$, a process that would require an

163 input of energy from either the flagellar ATPase and/or the proton motive force. We reasoned that the subunit 'gate opening' signal must somehow be transmitted to FlhA, triggering it to energise opening of the export gate. Previous studies have

166 shown that, in addition, interaction of FlhA with the FliJ stalk component of the

167 ATPase is required to convert the fT3SS into a highly efficient $\Delta \psi$ driven export

168 machine, suggesting that the FliJ-FlhA interaction might be a second signal required 169 for export gate opening ${ }^{17}$. Loss of either the FliJ-FlhA interaction or the subunit N- 
terminal hydrophobic signal can be overcome by suppressor mutations in the export

171 machinery: FliH-Flil-FliJ loss can be overcome by mutations in FlhA or FlhB ${ }^{23,24}$, and

172 we have shown that loss of the interaction between the FlgD short subunit N-terminal hydrophobic signal and the export machinery can be overcome by mutations in the

174 genes encoding FliP or FliR that destabilise the export gate closed conformation ${ }^{19}$.

175 We now wanted to test whether the suppressor mutations in the ABPQR export gate

176 (FliR-F $\mathrm{F}_{113} \mathrm{~A}$ or FliR-G $\left.{ }_{117} \mathrm{D}\right)$ that recovered export of FlgD $\mathrm{D}_{\text {short }}$ could also recover

177 motility and export in strains deleted for the ATPase genes (in which the FliJ-FlhA is

178 lost; Fig. 4A and 4B). To test this, we constructed strains in which the chromosomal

179 fliR gene was replaced with fliR alleles containing the gate opening mutations $\left(\mathrm{F}_{113} \mathrm{~A}\right.$

180 or $\mathrm{G}_{117} \mathrm{D}$ ) in combination with deletions in the genes that encode the ATPase

181 components FliH and Flil. These strains were also deleted for the gene encoding the

182 flagellar anti-sigma factor FlgM, which is exported during the filament stage of assembly ${ }^{25}$. Deletion of flgM ensures that flagellar gene expression is not influenced by any differences in export efficiency between strains ${ }^{26}$ (Fig. 4). Mutations that promoted the open gate conformation $\left(F l i R-F_{113} A\right.$ or FliR- $\left.G_{117} D\right)$ did not recover motility and export in strains deleted for the ATPase complex (Fig. 4A and 4B). This

187 indicates that mutations that promote the open conformation of the FliPQR gate cannot bypass the loss of FliJ-dependent activation of FlhA.

190 of flagellar ATPase activity (and, as a result, bypass of the FliJ-FlhA interaction), 191 could recover efficient gate opening by $\mathrm{FlgD}_{\text {short }}$ (Fig. 4C). A Salmonella flgD null 192 strain encoding $f / h B-\mathrm{P}_{28} \mathrm{~T}$ and producing FlgD $\mathrm{D}_{\text {short }}$ was found to be no more motile 193 than the parental Salmonella flgD null strain producing FlgD short (Fig 4C and S5). 
194 This indicates that the FlhB- $\mathrm{P}_{28}$ T mutation does not promote opening of the export

195 gate, but instead restores fT3SS activity in the absence of the FliJ activation signal

196 by an alternative mechanism (Fig. 4C).

These data indicate that mutations in fliP or fliR that promote the open

198 conformation of the export gate and mutations that bypass the need for the FliJ-FlhA

199 interaction are unable to compensate for each other, indicating that the subunit N-

200 terminal hydrophobic export signal and the FliJ-FlhA interaction signal are non-

201 redundant, i.e. both signals are required to activate pmf-driven opening of the

202 ABPQR export gate by FlhA (Fig. 5).

\section{Discussion}

Transport of substances across the bacterial inner membrane is highly

selective, thereby maintaining the essential electrochemical gradients that drive

numerous processes at the cell membrane, including ATP synthesis and protein

export. The fT3SS translocates proteins efficiently across the inner membrane

without compromising the permeability barrier ${ }^{5,18,21}$. The ABPQR flagellar export gate

contains three constriction points that are proposed to maintain the gate in a closed

211 conformation, preserving the integrity of the membrane permeability barrier ${ }^{5}$. Here,

we identified point mutant and deletion variants of proteins in the ABPQR export gate

213 that destabilise its closed conformation. We show that either by destabilising the

214 export gate closed conformation or by increasing the pmf, the flagellar export defects

215 of strains encoding FlhA- $\mathrm{K}_{203} \mathrm{~A}$ can be alleviated, indicating that FlhA harnesses the

216 pmf to energise opening of the export gate complex. Additional in vivo experiments

217 revealed that FIhA requires activation by both the flagellar ATPase stalk component 
218 FliJ and an N-terminal hydrophobic signal in the export substrate to trigger efficient

219 gate opening and subunit export.

220

Mutational analysis of the FliR-plug identifies variants that destabilise the

closed conformation of the export gate.

Structures of the FliPQR export gate have revealed that, in the absence of the

export substrate, multiple non-covalent interactions in the gate complex stabilise the

closed conformation ${ }^{5,12,18}$. In particular, a 15-residue region of FliR forms a plug that

226

occludes the central channel of the gate. Two residues within the FliR-plug, FliR-F $F_{113}$

227

and FliR-F $\mathrm{F}_{115}$, interact with a concentric ring of conserved methionine residues (M-

228

gate) in FliP5. Our mutational analyses identified FliR variants, FliR-F $\mathrm{F}_{113} \mathrm{~A}$ and FliR-

229

$F_{115} \mathrm{~A}$, that destabilise the closed conformation of the export gate ${ }^{5}$, indicating that

230 these residues help to maintain the gate in its closed state in the absence of export

substrate. An additional FliR-plug variant, FliR-G ${ }_{117} \mathrm{D}$, also destabilised the closed

232

gate, suggesting that the introduction of a bulky, charged side chain at this site

233

prevents tight closure of the gate. A recent structure of the open export gate

revealed multiple rearrangements within the M-gate and an upward displacement of

the FliR plug to allow subunit passage ${ }^{18}$. This indicates that the FliR plug, which

occludes the export channel in the closed gate conformation, must be displaced to

allow passage of unfolded subunits through the gate. A strain containing a deletion

within the FliR-plug (residues 110-116) displayed choline sensitivity, consistent with

the plug forming a tight seal to maintain the membrane permeability barrier (Fig 1).

Notably, we found that a FliR-plug deletion strain that produced fewer subunits was 
242 produced fewer subunits (Fig 1). These data indicate that (i) subunit transit through

243 the export gate can partially rescue the choline-sensitive phenotype associated with

244 loss of the FliR-plug, and (ii) that when no subunits available for transit, the wild type

245 export gate closes to maintain the membrane permeability barrier. These

246 observations have important implications for our understanding of how the export

247 gate opens. Sequential rounds of gate opening and closing must occur in response

248 to the presence or absence of export substrates at the membrane export machinery.

249 Similar gating mechanisms have been observed in other protein conducting

250 channels, such as the SecY channel, which similarly contains a constriction formed

251 by ring of hydrophobic residues and a central plug that forms a seal ${ }^{27}$. Deletion of

252 either the central plug or the ring of hydrophobic residues in SecY results in

253 increased permeability of the channel, and electrophysiology experiments showed

254 that these deletions cause the channel to alternate between an open and closed state $^{28}$. Furthermore, molecular dynamic simulations indicate that the force required for opening the gate is reduced in SecY plug deletion mutants ${ }^{29}$. We found that deletion of the FliR plug similarly destabilised the closed conformation of the export gate and concomitantly increased permeability of the inner membrane (Fig. 1).

FIhA activity is required for efficient opening of the export gate.

A previous study identified suppressor mutations in the gene encoding FliR

262 that rescued the export defect of a strain that contained mutations in $f / h A\left(\mathrm{~K}_{203} \mathrm{~W}\right)$ and $f / h B\left(\mathrm{P}_{28} \mathrm{~T}\right)$, as well as deletions in the genes encoding flagellar export ATPase components (fliH and fli ${ }^{20}$. The authors proposed that the FliR suppressor mutations might recover an interaction between FliR and $\mathrm{FlhA}^{20}$. One of these 
suppressor mutations (FliR-G $\mathrm{G}_{117} \mathrm{D}$ ) is positioned within the core of the FliPQR

267 complex and introduces a bulky, charged side chain into the lumen of the export

268 gate. We showed that the FliR-G ${ }_{117} \mathrm{D}$ mutation partially suppressed the motility

269 defect associated with a subunit ( $\left.F l g D_{\text {short }}\right)$ that is unable to trigger efficient opening

270 of the export gate, indicating that the $F\left(i R-G_{117} D\right.$ mutation might instead destabilise

271 the export gate's closed conformation (Fig. 1B and 1C). We hypothesised that by the

272 same mechanism, the FliR-G ${ }_{117} \mathrm{D}$ mutation might also suppress the motility and

273 export defects associated with FlhA-K 203 . Salmonella strains carrying the flhA-K $\mathrm{K}_{203} \mathrm{~A}$

274 mutation in combination with mutations in fliR $\left(\mathrm{F}_{113} \mathrm{~A}\right.$ or $\left.\mathrm{G}_{117} \mathrm{D}\right)$, which destabilise the

275 closed conformation of the export gate, displayed enhanced flagellar export and

276 motility compared to the parental Salmonella flhA-K $203 \mathrm{~A}$ strain (Fig. 2). This indicates

277 that FlhA- $\mathrm{K}_{203} \mathrm{~A}$ is unable to facilitate efficient export gate opening and, as a result,

278 attenuates subunit export and cell motility.

Cryo-electron tomograms of the Salmonella injectisome have revealed that

the FlhA homologue, InvA, is positioned adjacent to the homologues of the FliPQR

281

export gate components, SpaPQR ${ }^{13}$. As FlhA is proposed to function as a proton

conducting channel that energises export, we reasoned proton translocation might

be coupled to gate opening, possibly through transmission of conformational

changes in FlhA to the adjacent FliPQR-FlhB components of the export gate ${ }^{13,14,20}$.

In support of this view, we found that by increasing the pmf across the inner

membrane, through supplementation of cell cultures with arginine, subunit export by

the $f / h A-K_{203} A$ strain could be enhanced by $40 \%$, suggesting that inefficient export in 
Two distinct signals activate the pmf-driven export machinery.

292 ATPase interacts with FlhA to convert the export machinery into a highly efficient $\Delta \Psi$ -

293 driven export machine ${ }^{17,30,31,32}$. The FliH and Flil components of the ATPase facilitate

294 the FliJ-FlhA interaction to promote full activation of the export gate ${ }^{17,31}$. These

295 findings indicate that FliJ binding to FlhA modulates its proton conducting ability,

296 analogous to the interactions between the stalk component of the $F_{1}$ ATPase and the

297 membrane-localised $F_{0}$ channel that drive proton translocation across the bacterial

298 inner membrane. The need for activation of FlhA by the FliJ stalk, to facilitate

299 efficient use of the $\Delta \psi$ component of the pmf and energise gate opening, can be

300 bypassed by mutations in $f / h A$ and $f l h B$ that overcome the loss of the flagellar

301 ATPase ${ }^{8,23,24}$.

302 In addition to the FilJ-dependent gate opening signal, we have previously

303 shown that a subunit $\mathrm{N}$-terminal hydrophobic signal is required to trigger opening of

304 the export gate ${ }^{19}$ (Fig. 1C). To determine whether both signals were required to trigger opening of the export gate or whether the signals were functionally redundant, we asked if suppressor mutations in the ABPQR export gate (FliR- $\mathrm{F}_{113} \mathrm{~A}$ or FliR-

$307 \mathrm{G}_{117} \mathrm{D}$ ) that could compensate for loss of the subunit N-terminal hydrophobic signal could also overcome loss of the flagellar ATPase (and of the FilJ-dependent gate

309 opening signal). We found that neither FliR-F $F_{113} A$ nor FliR-G $G_{117} D$ could suppress the export defect caused by the loss of FliJ-dependent activation of FlhA (Fig.4). Taking

311 the reciprocal approach, we found that a mutation in $f / h B\left(\mathrm{P}_{28} \mathrm{~T}\right)$ that alleviates the

312 loss of the FliJ-dependent activation signal could not overcome loss of the subunit $\mathrm{N}$ 313 terminal hydrophobic signal (Fig 4). This indicates that both signals are essential for 
314 efficient opening of the flagellar export gate. We propose that FliJ binding to FlhA

315 and the presence of a subunit at the export machinery induce distinct conformational

316 changes in FlhA, rendering the export machinery competent to utilise the pmf to

317 energise opening of the export gate. The lack of either signal prevents opening of the

318 export gate and, in turn, maintains the membrane permeability barrier (Fig. 5).

$319 \quad$ Based on the structural and functional similarities between the core

320 components of the injectosome and flagellar Type III Secretion Systems, it is highly

321 probable that the mechanism of export gate opening is conserved. In summary, we

322 propose that the pmf induces conformational changes in FlhA that are transmitted to

323 the adjacent FliPQR-FlhBN components of the export gate, energising gate opening.

324 We demonstrate that FlhA requires at least two distinct signals to facilitate subunit

325 export: a FliJ-dependent activation signal and a subunit docked at the export

326 machinery. Our data suggest a multi-signal control system that acts via FlhA to

327 ensure that use of the pmf and export gate opening only occurs when a FliJ

328 activation signal is received and a subunit is available for export.

329

$330 \quad$ Materials and Methods

331 Bacterial strains, plasmids and growth conditions

332 Salmonella strains and plasmids used in this study are listed in table 1. The

$333 \Delta f l g D:: \mathrm{K}_{\mathrm{m}}^{\mathrm{R}}$ strain in which the flgD gene was replaced by a kanamycin resistance

334 cassette and the $\triangle f l g B C D E F G H I J:: \mathrm{Km}^{\mathrm{R}}$ strain in which a large portion of the flg

335 operon was replaced with a kanamycin cassette were constructed using the $\lambda$ Red

336 recombinase system ${ }^{33,34}$. Strains containing chromosomally encoded FliR and/or

337 FlhA variants were constructed by aph-I-Scel Kanamycin resistance cassette 
replacement using pWRG73034. Recombinant proteins were expressed in

339 Salmonella from isopropyl $\beta$-D-thiogalactoside-inducible (IPTG) inducible plasmid

$340 \mathrm{pTrc} 9 \mathrm{a}^{35}$. Bacteria were cultured at $30-37^{\circ} \mathrm{C}$ in Luria-Bertani (LB) broth containing

341 ampicillin $(100 \mu \mathrm{g} / \mathrm{ml})$.

\section{Choline sensitivity assay}

344 The choline sensitivity assay was performed essentially as described in Ward et

345 al. ${ }^{21}$. Cells were grown overnight at $32^{\circ} \mathrm{C}$ in terrific broth (TB) medium with shaking

346 (200 PRM). The overnight cultures were diluted 1:50 in TB and grown at $32^{\circ} \mathrm{C}$ with

347 shaking (200 RPM) to an A600 1.0. These cultures were diluted 1:50 in TB

348 containing choline at concentrations as indicated in Figure 2. The cultures were

349 grown for 4.5 hours at $32^{\circ} \mathrm{C}$ with shaking (200 RPM) and the A600 measured. The

350 Miles and Misra method ${ }^{36}$ was used to determine the colony forming units for strains

351 grown in $300 \mathrm{mM}$ choline. 10-fold dilutions of culture were spotted (10 $\mu \mathrm{l})$ onto LB

352 agar plates and grown overnight at $37^{\circ} \mathrm{C}$.

\section{Flagellar subunit export assay}

355 Salmonella strains were cultured at $37^{\circ} \mathrm{C}$ in LB broth containing ampicillin and IPTG

356 to mid-log phase (OD $600 \mathrm{~nm}$ 0.6-0.8). Cells were centrifuged $(6000 \mathrm{~g}, 3 \mathrm{~min})$ and

357 resuspended in fresh media and grown for a further 60 minutes at $37^{\circ} \mathrm{C}$. Cells were

358 pelleted by centrifugation $(16,000 \mathrm{~g}, 5 \mathrm{~min})$ and the supernatant passed through a 0.2

$359 \mu \mathrm{m}$ nitrocellulose filter. Proteins were precipitated with $10 \%$ trichloroacetic acid

360 (TCA) and 1\% Triton-X100 on ice for 1 hour, pelleted by centrifugation $(16,000 \mathrm{~g}, 10$

$361 \mathrm{~min}$ ), washed with ice-cold acetone and resuspended in SDS-PAGE loading buffer 
362 (volumes calibrated according to cell densities). Fractions were analysed by

363 immunoblotting. For the export assays of strains supplemented with arginine, cells

364 were treated essentially as described above except cells were resuspended in fresh

365 media supplemented with $20 \mathrm{mM}$ arginine and grown for a further 30 minutes instead

366 of 60 minutes.

\section{Motility assays}

368 For swimming motility, cultures were grown in LB broth to A600nm 1. Two microliters

369 of culture were inoculated into soft tryptone agar $(0.3 \%$ agar, $10 \mathrm{~g} / \mathrm{L}$ tryptone, $5 \mathrm{~g} / \mathrm{L}$

$370 \mathrm{NaCl})$ containing ampicillin $(100 \mu \mathrm{g} / \mathrm{ml})$. Plates were incubated at $37^{\circ} \mathrm{C}$ for between

3714 and 6 hours unless otherwise stated. For swarming motility, one microliter of

372 overnight culture grown in LB broth was inoculated onto tryptone agar plates $(0.6 \%$

373 agar, $1 \% \mathrm{w} / \mathrm{v}$ tryptone, $0.5 \% \mathrm{w} / \mathrm{v}$ sodium chloride) containing appropriate antibiotics

374 and inducing agents and supplemented with $0.3 \%$ glucose and incubated at $30{ }^{\circ} \mathrm{C}$

375 for $12-16$ hours.

\section{Screen for motile suppressors from the fIhA-K203A variant}

378 Cells of the Salmonella flhA-K $\mathrm{K}_{203} \mathrm{~A}$ strain were cultured at $37^{\circ} \mathrm{C}$ in LB broth to mid-

379 log phase and inoculated into soft tryptone agar (0.3\% agar, $10 \mathrm{~g} / \mathrm{L}$ tryptone, $5 \mathrm{~g} / \mathrm{L}$

$380 \mathrm{NaCl}$ ). Plates were incubated at $30^{\circ} \mathrm{C}$ until motile 'spurs' appeared. Cells from the

381 spurs were streaked to single colony and the flhA gene sequenced. New strains

382 containing the $f / h A-K_{203} A$ and suppressor mutations were constructed by aph-I-Scel

$383 \lambda$ Red recombination to confirm that the mutations within $f / h A$ were responsible for

384 the motility suppressor phenotype ${ }^{34}$. 


\section{Quantification and statistical analysis}

387 Experiments were performed at least three times. Immunoblot were quantified using

388 Image Studio Lite. The unpaired two-tailed Student's $t$-test was used to determine $p$ -

389 values and significance was determined as ${ }^{*} p<0.05$. Data are represented as mean

$390 \pm$ standard error of the mean (SEM), unless otherwise specified and reported as

391 biological replicates.

392

393 Author contributions

394 O.J.B and G.M.F conceived and designed experiments. O.J.B conducted

395 experiments. O.J.B analysed the data. O.J.B and G.M.F wrote the paper

396

397 Competing interests

398 The authors declare no competing interests.

399

400 Acknowledgements

401 This work was funded by a grant from the Biotechnology and Biological Sciences

402 Research Council (BB/M007197/1) to G.M.F, and a University of Cambridge John

403 Lucas Walker studentship to O.J.B.

404

405

Materials \& Correspondence

406 Materials are available from the corresponding authors upon request.

407

408

409 
412 1. Deng, W. et al. Assembly, structure, function and regulation of type III secretion systems. Nature Reviews Microbiology (2017). doi:10.1038/nrmicro.2017.20

2. Galán, J. E., Lara-Tejero, M., Marlovits, T. C. \& Wagner, S. Bacterial Type III Secretion Systems: Specialized Nanomachines for Protein Delivery into Target Cells. Annu. Rev. Microbiol. (2014). doi:10.1146/annurev-micro-092412155725

3. Evans, L. D. B., Hughes, C. \& Fraser, G. M. Building a flagellum outside the bacterial cell. Trends in Microbiology (2014). doi:10.1016/j.tim.2014.05.009 outer space. Microb. cell (Graz, Austria) (2014).

5. Kuhlen, L. et al. Structure of the core of the type iii secretion system export apparatus. Nat. Struct. Mol. Biol. (2018). doi:10.1038/s41594-018-0086-9

6. Johnson, S., Kuhlen, L., Deme, J. C., Abrusci, P. \& Lea, S. M. The Structure of an Injectisome Export Gate Demonstrates Conservation of Architecture in the Core Export Gate between Flagellar and Virulence Type III Secretion Systems. MBio (2019). doi:10.1128/mbio.00818-19

7. Abrusci, P. et al. Architecture of the major component of the type III secretion system export apparatus. Nat. Struct. Mol. Biol. 20, 99-104 (2012).

8. Saijo-Hamano, Y. et al. Structure of the cytoplasmic domain of FlhA and (2010). 
434 9. Zarivach, R., Vuckovic, M., Deng, W., Finlay, B. B. \& Strynadka, N. C. J.

Structural analysis of a prototypical ATPase from the type III secretion system.

Nat. Struct. Mol. Biol. 14, 131-137 (2007).

437

10. Imada, K., Minamino, T., Tahara, A. \& Namba, K. Structural similarity between the flagellar type III ATPase Flil and F1-ATPase subunits. Proc. Natl. Acad.

Sci. U. S. A. 104, 485-90 (2007).

11. Hu, J. et al. T3S injectisome needle complex structures in four distinct states reveal the basis of membrane coupling and assembly. Nat. Microbiol. (2019).

12. Kuhlen, L. et al. The substrate specificity switch FlhB assembles onto the export gate to regulate type three secretion. Nat. Commun. (2020). doi:10.1038/s41467-020-15071-9

13. Butan, C., Lara-Tejero, M., Li, W., Liu, J. \& Galán, J. E. High-resolution view of the type III secretion export apparatus in situ reveals membrane remodeling and a secretion pathway. Proc. Natl. Acad. Sci. U. S. A. (2019). doi:10.1073/pnas.1916331116

14. Erhardt, M. et al. Mechanism of type-III protein secretion: Regulation of FlhA conformation by a functionally critical charged-residue cluster. Mol. Microbiol. (2017). doi:10.1111/mmi.13623

15. Ibuki, T. et al. Common architecture of the flagellar type III protein export apparatus and F- and V-type ATPases. Nat. Struct. Mol. Biol. 18, 277-282 (2011).

16. Kishikawa, J. ichi et al. Common Evolutionary Origin for the Rotor Domain of 
17. Minamino, T., Morimoto, Y. V., Hara, N. \& Namba, K. An energy transduction mechanism used in bacterial flagellar type III protein export. Nat. Commun. (2011). doi:10.1038/ncomms1488

18. Sean Miletic, Dirk Fahrenkamp, Nikolaus Goessweiner-Mohr, Jiri Wald, Maurice Pantel, Oliver Vesper, Vadim Kotov, and T. C. M. Substrate-engaged type III secretion system structures reveal gating mechanism for unfolded protein translocation. bioRxiv (2020).

19. Owain J. Bryant, Paraminder Dhillon, Colin Hughes, G. M. F. Sequential recognition of discrete export signals in flagellar subunits during bacterial Type III secretion. bioRxiv (2020).

20. Hara, N., Namba, K. \& Minamino, T. Genetic characterization of conserved charged residues in the bacterial flagellar type III export protein FlhA. PLoS One 6, (2011).

21. Ward, E. et al. Type-III secretion pore formed by flagellar protein FliP. Mol. Microbiol. (2018). doi:10.1111/mmi.13870

22. Armbruster, C. E., Hodges, S. A., Smith, S. N., Alteri, C. J. \& Mobley, H. L. T. Arginine promotes Proteus mirabilis motility and fitness by contributing to conservation of the proton gradient and proton motive force. Microbiologyopen (2014). doi:10.1002/mbo3.194

23. Minamino, T., González-Pedrajo, B., Kihara, M., Namba, K. \& Macnab, R. M. The ATPase Flil can interact with the type III flagellar protein export apparatus in the absence of its regulator, FliH. J. Bacteriol. (2003). doi:10.1128/JB.185.13.3983-3988.2003

24. Minamino, T. \& Namba, K. Distinct roles of the Flil ATPase and proton motive 
force in bacterial flagellar protein export. Nature (2008).

25. Hughes, K. T., Gillen, K. L., Semon, M. J. \& Karlinsey, J. E. Sensing structural intermediates in bacterial flagellar assembly by export of a negative regulator. Science (80-. ). (1993). doi:10.1126/science.8235660

26. Chilcott, G. S. \& Hughes, K. T. Coupling of Flagellar Gene Expression to Flagellar Assembly in Salmonella enterica Serovar Typhimurium andEscherichia coli. Microbiol. Mol. Biol. Rev. (2000). doi:10.1128/mmbr.64.4.694-708.2000

27. Van Den Berg, B. et al. X-ray structure of a protein-conducting channel. Nature (2004). doi:10.1038/nature02218

28. Saparov, S. M. et al. Determining the Conductance of the SecY Protein Translocation Channel for Small Molecules. Mol. Cell (2007).

29. Gumbart, J. \& Schulten, K. The roles of pore ring and plug in the secY proteinconducting channel. J. Gen. Physiol. (2008). doi:10.1085/jgp.200810062

30. Minamino, T., Morimoto, Y. V., Hara, N., Aldridge, P. D. \& Namba, K. The doi:10.1371/journal.ppat.1005495

31. Minamino, T. et al. FliH and Flil ensure efficient energy coupling of flagellar type III protein export in Salmonella. Microbiologyopen (2016). 
subunit availability to activation of flagellar Type III Secretion. bioRxiv

33. Datsenko, K. A. \& Wanner, B. L. One-step inactivation of chromosomal genes in Escherichia coli K-12 using PCR products. Proc. Natl. Acad. Sci. U. S. A. (2000). doi:10.1073/pnas.120163297

34. Hoffmann, S., Schmidt, C., Walter, S., Bender, J. K. \& Gerlach, R. G. Scarless deletion of up to seven methylaccepting chemotaxis genes with an optimized method highlights key function of CheM in Salmonella Typhimurium. PLoS One (2017). doi:10.1371/journal.pone.0172630

514 35. Amann, E., Ochs, B. \& Abel, K. J. Tightly regulated tac promoter vectors useful for the expression of unfused and fused proteins in Escherichia coli. Gene (1988). doi:10.1016/0378-1119(88)90440-4

517 36. Miles, A. A., Misra, S. S. \& Irwin, J. O. The estimation of the bactericidal power of the blood. J. Hyg. (Lond). (1938). doi:10.1017/S002217240001158X 


\section{$521 \quad$ Figure legends}

\section{Figure 1 Mutations within the FliR plug destabilise the closed export gate}

524 a. Top view of the cryo-EM structure of the closed conformation of the FliPQR export

525 gate complex (PDB:6F2D). Phenylalanine residues 110 and 113 of FliR (grey) are

526 displayed in red. Methionine residue 210 of FliP (blue) is displayed in magneta. FliQ

527 is displayed in orange.

528 b. Swimming motility of recombinant Salmonella flgD null strains producing

529 chromosomally-encoded FliR variants $\left(F_{110} A, F_{113} A, F_{110} A+F_{113} A\right.$ or $\left.G_{117} D\right)$ or wild

530 type FliR (FliR). Strains produced a pTrc99a plasmid-encoded FlgD subunit variant

531 in which residues 9-32 were replaced with two repeats of the six amino acid

532 sequence Gly-Ser-Thr-Asn-Ala-Ser (FlgD short). Motility was assessed in $0.25 \%$ soft-

533 tryptone agar containing $100 \mu \mathrm{g} / \mathrm{ml}$ ampicillin and $50 \mu \mathrm{M}$ IPTG and incubated at

$53437^{\circ} \mathrm{C}$ for 16 hours.

535 c. The mean motility halo diameter of recombinant Salmonella flgD null strains

536 producing chromosomally-encoded FliR variants $\left(F_{110} A, F_{113} A, F_{110} A+F_{113} A\right.$ or

$\left.537 \mathrm{G}_{117} \mathrm{D}\right)$ or wild type FliR (FliR) and expressing the FlgDshort variant was plotted as a

538 percentage of the wild type FliR strain (FliR) producing FlgDshort (right hand bar).

539 Error bars represent the standard error of the mean calculated from at three

540 biological replicates. * indicates a $p$-value $<0.05$.

541 d. A cartoon schematic illustrating the effect of FliR plug mutations (red) on the

542 conformation of the export gate complex (blue). Data indicates that FliR plug

543 mutations destabilise the closed export gate conformation (left) and bias the open

544 conformation (right). 
546 e. Choline sensitivity of a Salmonella strain producing a chromosomally-encoded

547 FliR variant in which residues $110-116$ of the FliR plug is deleted ( $\Delta$ plug, grey), a

548 Salmonella strain deleted for the genes (flgBCDEFGHIJ) that encode most of the

549 early flagellar rod and hook subunits ( $\Delta$ subunits, orange), a Salmonella strain that

550 both produces the chromosomally-encoded FliR $\Delta$ plug variant and is deleted for the

551 genes ( $f l g B C D E F G H I$ ) that encode most of the early flagellar rod and hook subunits

552 ( $\Delta$ plug $\Delta$ subunits, blue) or a wild type Salmonella strain (FliR, yellow). Cells were

553 grown in Terrific broth containing varying concentrations of choline (as indicated) and

554 the optical density $\left(\mathrm{A}_{600}\right)$ measured following 4.5 hours.

555 f. Serial dilutions of the above Salmonella strains following 4.5 hours of growth in

556 Terrific broth supplemented with $300 \mathrm{mM}$ choline were spotted onto LB agar plates

557 using the Miles and Misra method. The strains are indicated on the left and the

558 density of the inoculum is indicated along the top.

559 g. A cartoon schematic illustrating the effect of the FliR plug deletion on the gating

560 activity of the export gate complex (blue). Strains that produce fewer subunits

561 ( $\Delta$ subunits and $\Delta$ plug $\Delta$ subunits) have fewer available subunits (red) for export to

562 seal the leaky phenotype of the export gate containing the FliR plug deletion $(\Delta$ plug,

563 right).

564

565 Figure 2 Mutations in the FliR plug suppress the FlhA-K 203 A motility and export defects

567 a. Whole cell (cell) and supernatant (secreted) proteins from late exponential-phase

568 cultures of a wild type Salmonella strain (wild type), a Salmonella strain producing a 
chromosomally-encoded FIhA- $\mathrm{K}_{203} \mathrm{~A}$ variant $\left(\mathrm{FlhA} \mathrm{K}_{203} \mathrm{~A}\right)$ or Salmonella strains

570 producing both a chromosomally-encoded FlhA-K $\mathrm{K}_{203} \mathrm{~A}$ variant and chromosomally

571 encoded FliR variants $\left(F_{113} A\right.$ or $\left.G_{117} D\right)$. Proteins were separated by SDS (15\%)-

572 PAGE and analysed by immunoblotting with anti-FliC, anti-FliD, anti-FlgK, anti-FlgD,

573 anti-FlhA or anti-FlgN polyclonal antisera. Apparent molecular weights are in

574 kilodaltons (kDa).

575 b. Swimming motility $(0.25 \%$ soft tryptone agar) of the same strains were carried out 576 at $37^{\circ} \mathrm{C}$ for $4-6$ hours.

577 c. Levels of FlgK in culture supernatants from Immunoblots were quantified using

578 ImageStudiolite and plotted as a percentage of FlgK exported by the wild type strain.

579 Error bars represent the standard error of the mean calculated from three biological 580 replicates. * indicates a $p$-value $<0.05$.

582 Figure 3 Increasing the proton-motive force suppresses the flhA-K $\mathrm{K}_{203} \mathrm{~A}$ export

\section{3 defect}

584 a. A cartoon schematic of a cell containing arginine decarboxylase enzyme which

585 catalyses the conversion of L-arginine into agmatine and carbon dioxide $\left(\mathrm{CO}_{2}\right)$. The

586 arginine decarboxylation reaction consumes a proton $\left(\mathrm{H}^{+}\right)$from the cell cytoplasm,

587 reducing the number of protons in the cell, effectively increasing the proton-motive

588 force.

589 b. Whole cell (cell) and supernatant (secreted) proteins from late exponential-phase

590 cultures of a wild type Salmonella strain (FlhA) or a Salmonella strain producing a

591 chromosomally-encoded FlhA- $\mathrm{K}_{203} \mathrm{~A}$ variant $\left(\mathrm{K}_{203} \mathrm{~A}\right)$. Cells were supplemented with

$592(+)$ or not supplemented with (-) 20 mM arginine 30 minutes prior to collection of 
whole cell and culture supernatants. Proteins were separated by SDS (15\%)-PAGE

594 and analysed by immunoblotting with anti-FlgK, anti-FlgD, anti-FlhA or anti-FlgN

595 polyclonal antisera (Left). Apparent molecular weights are in kilodaltons (kDa).

596 Levels of FlgK in culture supernatants from Immunoblots for the FlhA-K $\mathrm{K}_{203} \mathrm{~A}$ strain

597 were quantified using ImageStudioLite and plotted as a percentage of FlgK exported

598 by strains supplemented with arginine $(+)$ to strains not supplemented with arginine

599 (-) (right). Error bars represent the standard error of the mean calculated from three

600 biological replicates. * indicates a $p$-value $<0.05$.

601

602 Figure 4 Mutations in the FliR plug do not suppress export and motility defects 603 of strains deleted for the ATPase complex

604 a. Swimming motility of recombinant Salmonella strains deleted for the genes that

605 encode; the anti-sigma(28) factor (flgM), the flagellar ATPase negative regulator

$606(\mathrm{fliH})$ and the flagellar ATPase subunit (fli) and producing either a chromosomally-

607 encoded FlhB-P28 variant or producing chromosomally encoded wild type FliR (-) or

608 its variants $\left(F_{113} A\right.$ or $\left.G_{117} D\right)$. Motility was assessed in $0.25 \%$ soft-tryptone agar and

609 incubated at $37^{\circ} \mathrm{C}$ for $4-6$ hours.

610 b. Whole cell (cell) and supernatant (secreted) proteins from late exponential-phase

611 cultures of the same strains were separated by SDS (15\%)-PAGE and analysed by

612 immunoblotting with anti-FliD, anti-FlgD, anti-FlhA or anti-FlgN polyclonal antisera.

613 Apparent molecular weights are in kilodaltons (kDa).

614 c. Swimming motility of recombinant Salmonella flgD null strains producing

615 chromosomally-encoded wild type FlhB or its variants (FlhB- $\left.\mathrm{P}_{28} \mathrm{~T}\right)$. Both strains

616 produced a pTrc99a plasmid-encoded FlgD subunit variant in which residues 9-32 
617 were replaced with two repeats of the six amino acid sequence Gly-Ser-Thr-Asn-Ala-

618 Ser (FlgD short). Motility was assessed in $0.25 \%$ soft-tryptone agar containing 100

$619 \mu \mathrm{g} / \mathrm{ml}$ ampicillin and $50 \mu \mathrm{M}$ IPTG and incubated at $37^{\circ} \mathrm{C}$ for 16 hours.

621 Figure 6 Two mutually exclusive signals are required to activate the export machinery and trigger opening of the export gate.

623 A cartoon schematic illustrating the effect of either (i) the absence of a subunits

624 docked at the export machinery (middle, $\Delta$ subunit) or (ii) the absence of a FliJ-FlhA

625 interaction (right, $\Delta$ FliJ) (required to convert the export machinery into a highly

626 efficient $\Delta \psi$-driven export machine) on the ability of the export machinery to energise

627 export gate opening (blue). ATP hydrolysis by the Flil (orange, PDB: 2DPY)

628 component of the ATPase complex is thought to drive rotation of the FliJ stalk

629 subunit (orange, PDB: 3AJW), allowing FliJ to bind all nine binding sites on the

630 nonameric ring of FIhA (green, PDB: 3A5I), converting the export machinery into a

631 highly efficient $\Delta \psi$-driven export machine. Early flagellar subunits dock at FlhBc (red,

632 PDB: 3B0Z) and contain a N-terminal hydrophobic export signal required for efficient

633 subunit export and translocation through the export gate complex (left). The absence

634 of subunits at the export machinery (middle) or the absence of FliJ-FlhA interactions

635 (right) renders the export machinery unable to utilise the PMF to drive opening of the 636 export gate.

637

638 


\section{Supplementary Figures}

\section{Figure S1}

643 Whole cell (cell) proteins from late exponential-phase cultures of recombinant

644 Salmonella flgD null strains producing chromosomally-encoded $F$ liR variants $\left(F_{110} A\right.$,

$645 \mathrm{~F}_{113} \mathrm{~A}, \mathrm{~F}_{110} \mathrm{~A}+\mathrm{F}_{113} \mathrm{~A}$ or $\left.\mathrm{G}_{117} \mathrm{D}\right)$ or wild type FliR (FliR), and a pTrc99a plasmid-

646 encoded FlgD subunit variant in which residues 9-32 were replaced with two repeats

647 of the six amino acid sequence Gly-Ser-Thr-Asn-Ala-Ser (FlgDshort). Proteins were separated by SDS (15\%)-PAGE and analysed by immunoblotting with anti-FlgD

649 polyclonal antisera. Apparent molecular weights are in kilodaltons (kDa).

\section{$651 \quad$ Figure S2}

652 A. Swimming (top panel; $0.25 \%$ soft tryptone agar) and swarming (bottom panels;

$6530.6 \%$ agar-tryptone with $0.5 \%$ glucose) motility of recombinant Salmonella strains 654 producing chromosomally-encoded FliR variants $\left(F_{110} A, F_{113} A, G_{117} D\right.$ or $\left.655 \quad F_{110} A+F_{113} A\right)$ or SJW1103 wild type.

B. Whole cell (cell) and secreted (secreted) proteins from late exponential-phase

657 cultures of recombinant Salmonella strains producing chromosomally-encoded FliR 658 variants $\left(F_{110} A, F_{113} A, G_{117} D\right.$ or $\left.F_{110} A+F_{113} A\right)$ or SJW1103 wild type. Proteins were separated by SDS (15\%)-PAGE and analysed by immunoblotting with anti-FliC, anti-

660 FliD, anti-FlgK, anti-FlgD, anti-FlhA and anti-FlgN polyclonal antisera. Apparent 661 molecular weights are in kilodaltons (kDa). 


\section{Figure S3}

665 Whole cell (cell) proteins from late exponential-phase cultures of recombinant

666 Salmonella strains producing a chromosomally-encoded FliR variant in which

667 residues $110-116$ of the FliR plug is deleted ( $\Delta$ plug), a Salmonella strain deleted for

668 the genes ( $f / g B C D E F G H I J)$ that encode most of the early flagellar rod and hook

669 subunits ( $\Delta$ subunits), a Salmonella strain that both produces the chromosomally-

670 encoded FliR $\triangle$ plug variant and is deleted for the genes (flgBCDEFGHIJ) that encode

671 most of the early flagellar rod and hook subunits ( $\Delta$ plug $\Delta$ subunits) and a SJW1103

672 wild type strain (FliR) were separated by SDS (15\%)-PAGE and analysed by

673 immunoblotting with anti-FlhA and anti-FlgD polyclonal antisera. Apparent molecular 674 weights are in kilodaltons (kDa).

$676 \quad$ Figure S4

677 Whole cell (cell) and supernatant (secreted) proteins from late exponential-phase

678 cultures of a strain deleted for two genes that encode arginine decarboxylase enzymes $(\triangle s p e A$ and $\Delta$ adi $A$, labelled FlhA) or a Salmonella $\Delta s p e A-\triangle$ adi $A$ strain

680 producing a chromosomally-encoded FlhA- $\mathrm{K}_{203} \mathrm{~A}$ variant $\left(\mathrm{K}_{203} \mathrm{~A}\right)$. Cells were

681 supplemented with (+) or not supplemented with (-) $20 \mathrm{mM}$ arginine 30 minutes prior 682 to collection of whole cell and culture supernatants. Proteins were separated by SDS 683 (15\%)-PAGE and analysed by immunoblotting with anti-FlgK, anti-FlgD, anti-FlhA or 684 anti-FlgN polyclonal antisera. Apparent molecular weights are in kilodaltons (kDa). 


\section{$688 \quad$ Figure S5}

689 Whole cell (cell) proteins from late exponential-phase cultures of recombinant

690 Salmonella flgD null strains producing chromosomally-encoded wild type FlhB or its

691 variants (FlhB-P $28 \mathrm{~T})$. Both strains produced a pTrc99a plasmid-encoded FlgD

692 subunit variant in which residues 9-32 were replaced with two repeats of the six

693 amino acid sequence Gly-Ser-Thr-Asn-Ala-Ser (FlgD short). Proteins were separated

694 by SDS (15\%)-PAGE and analysed by immunoblotting with anti-FlgD polyclonal

695 antisera. Apparent molecular weights are in kilodaltons (kDa). 
a

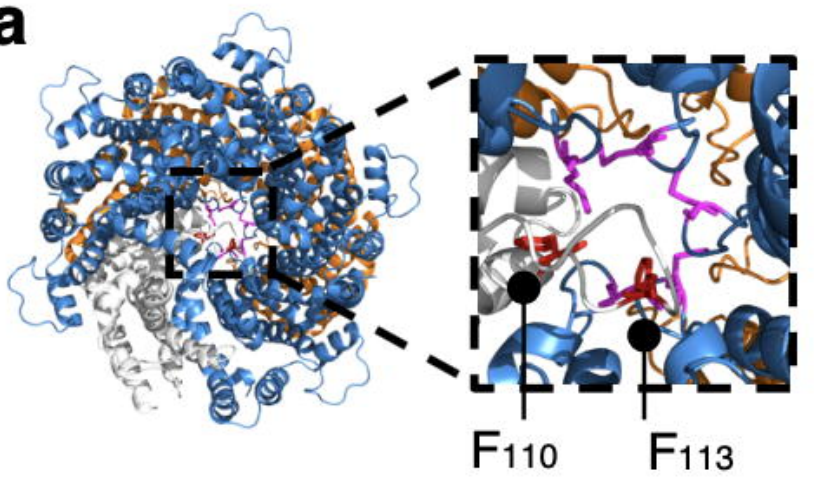

b

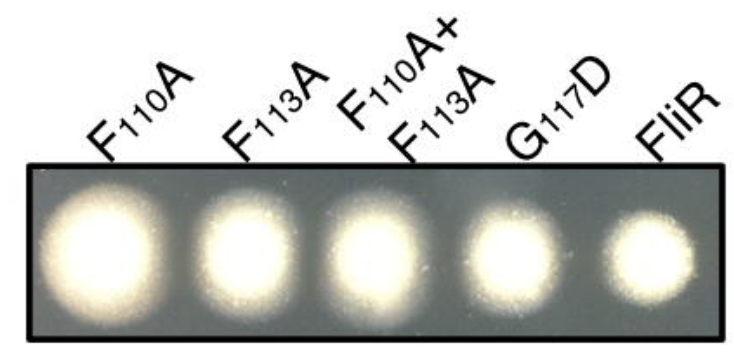

FliR mutation, $\Delta f l g D+$ FlgDshort

C

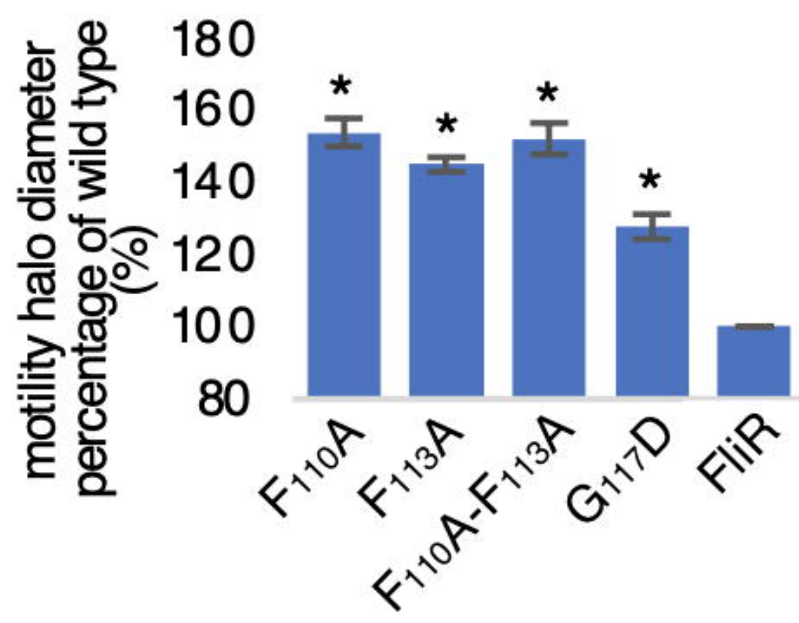

d

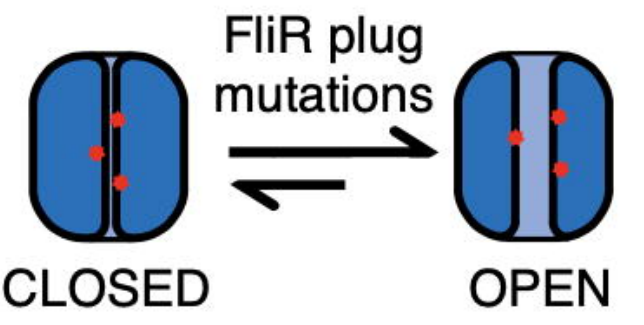

e

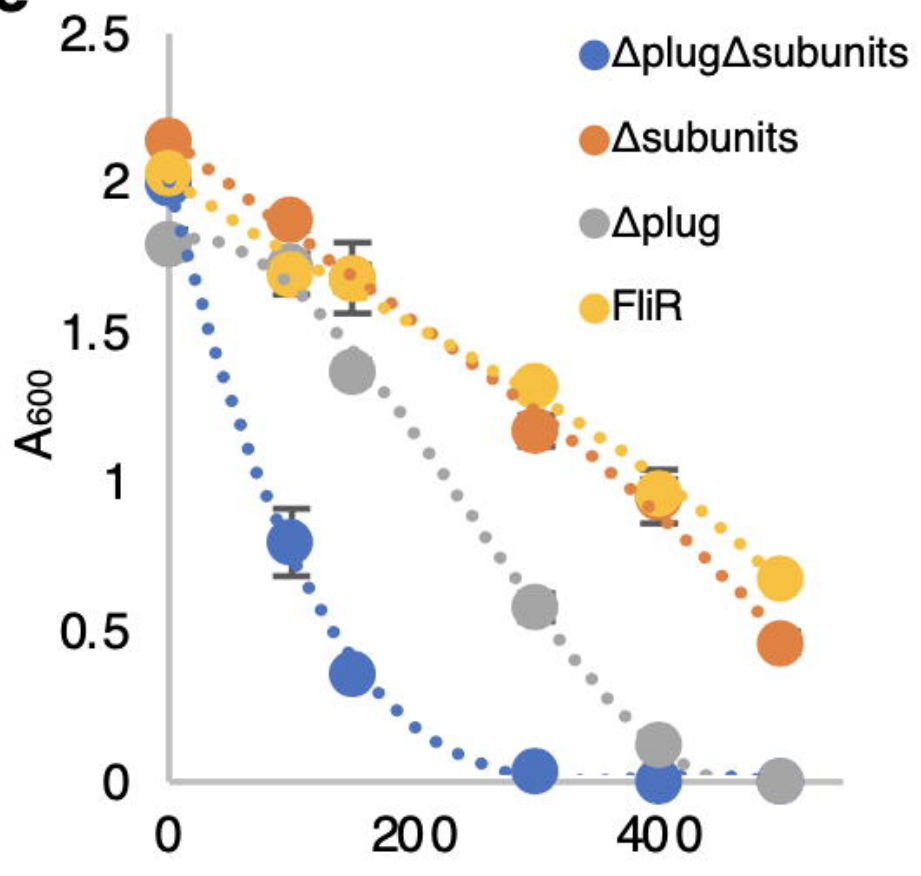

Choline (mM)
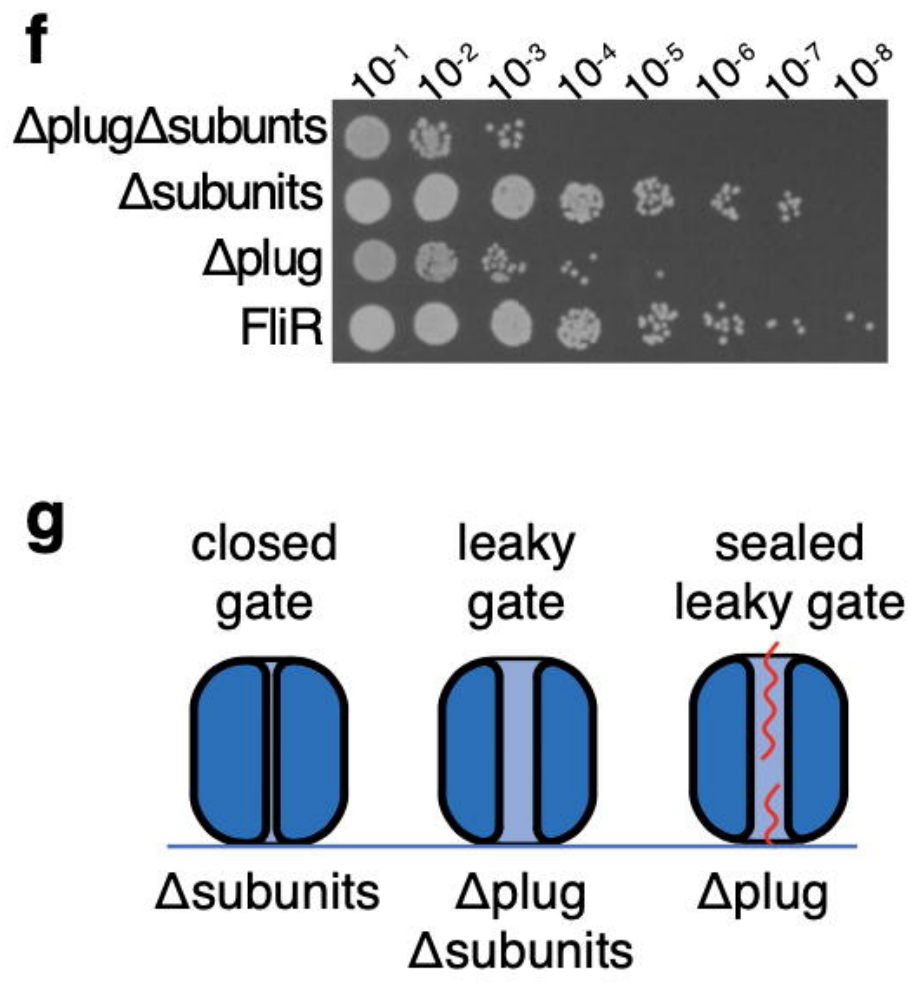
a

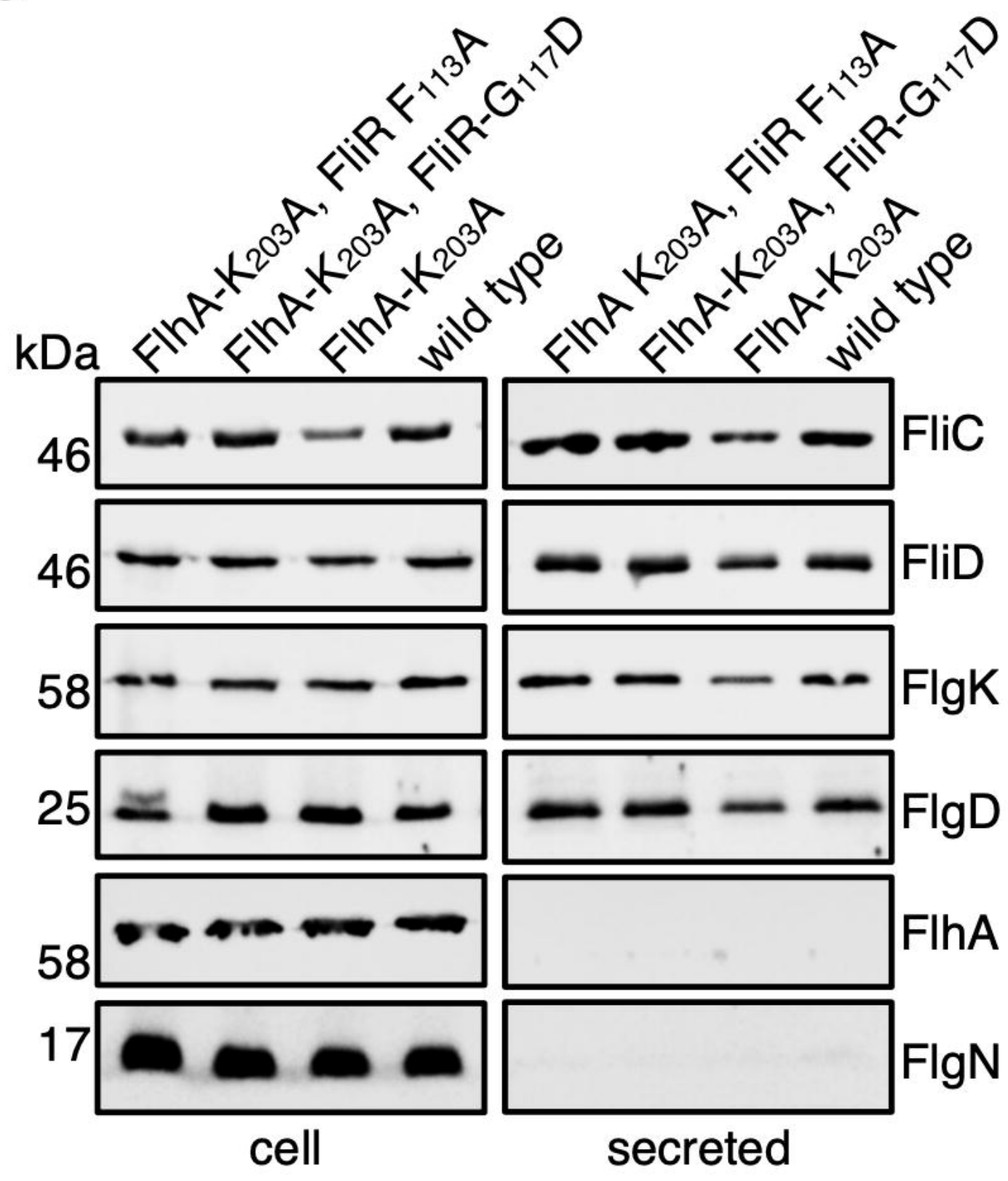

b

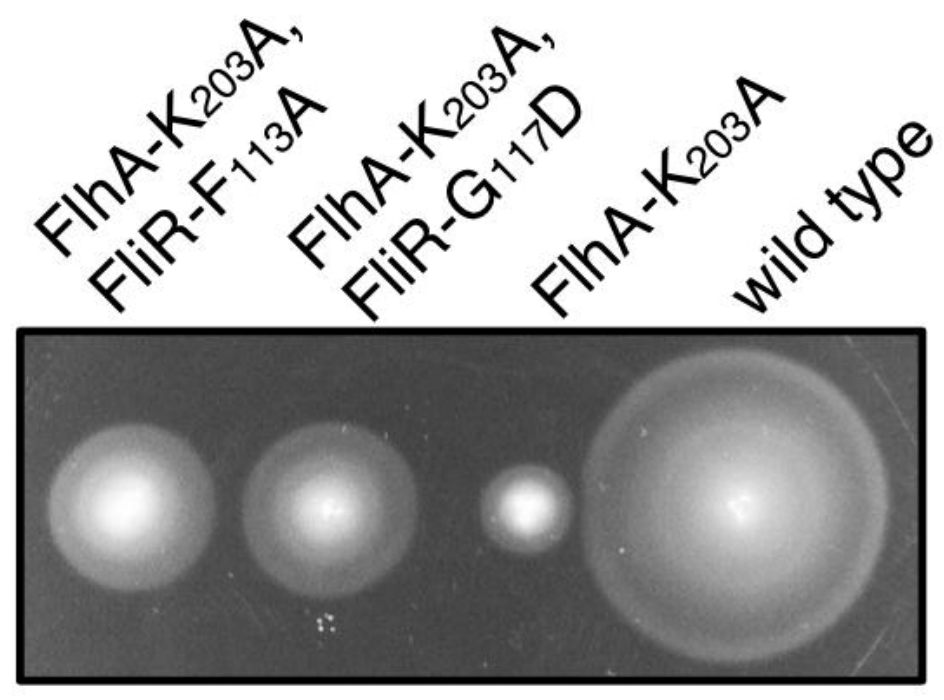

c

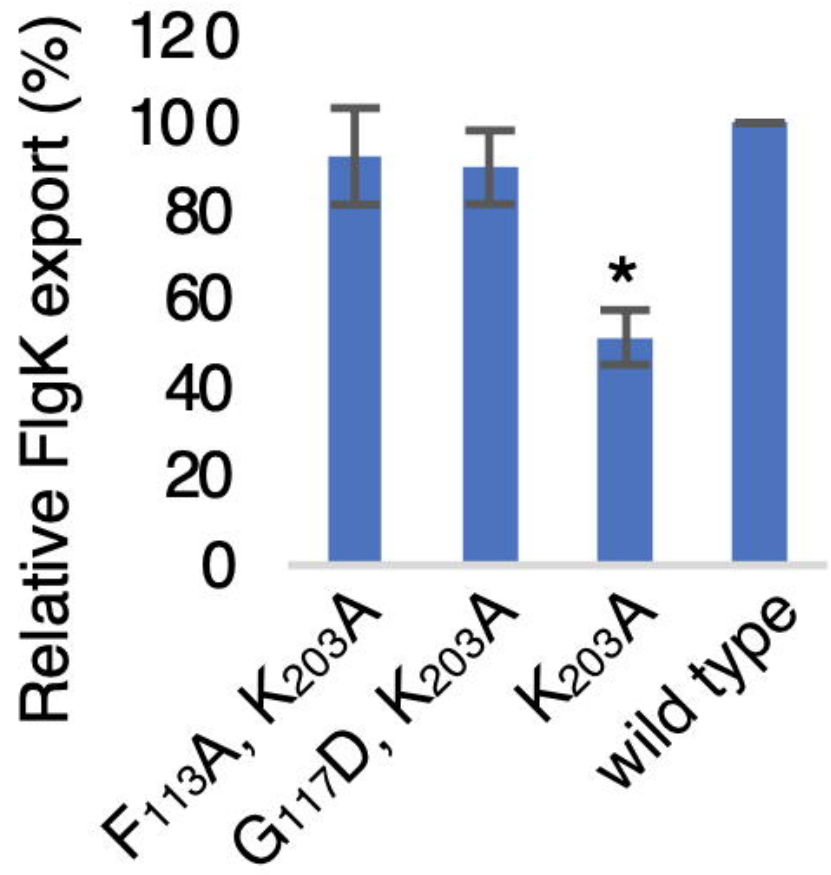

Figure 2 
a

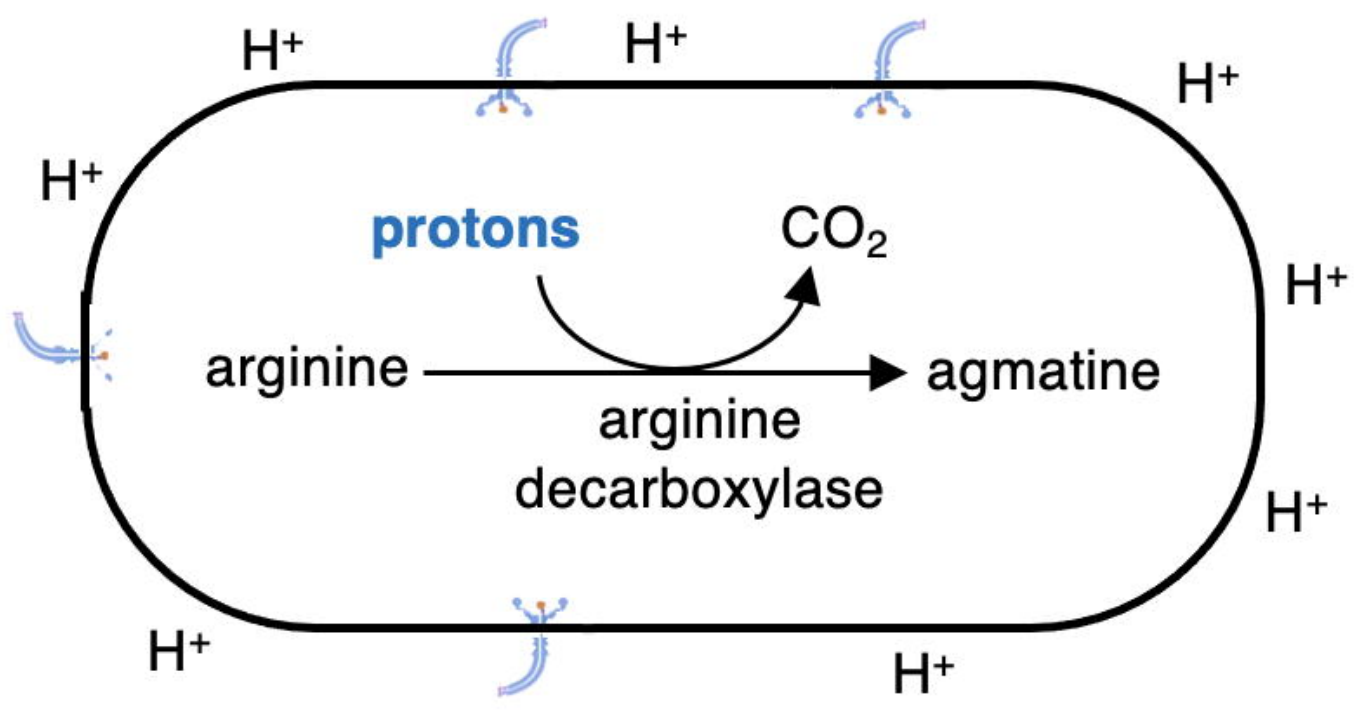

b

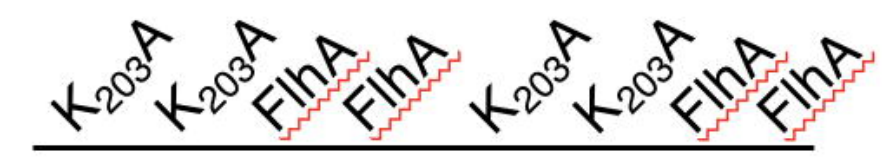

$\mathrm{kDa}_{+}++\quad+\quad+\quad+\quad-$ arginine

58

$-\infty$

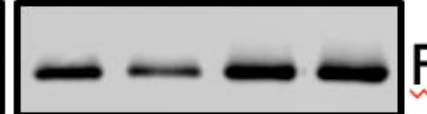

FlaK
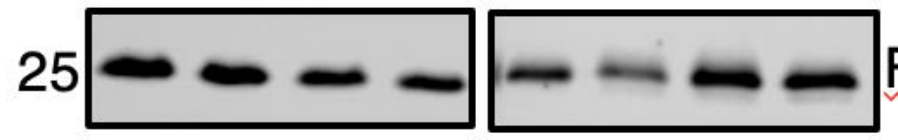

FlgD

$58-\infty-$

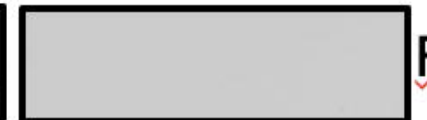

FlhA

17

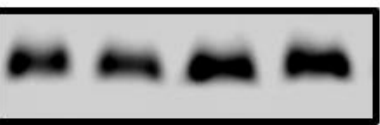

cell

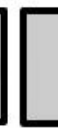

FlgN

secreted

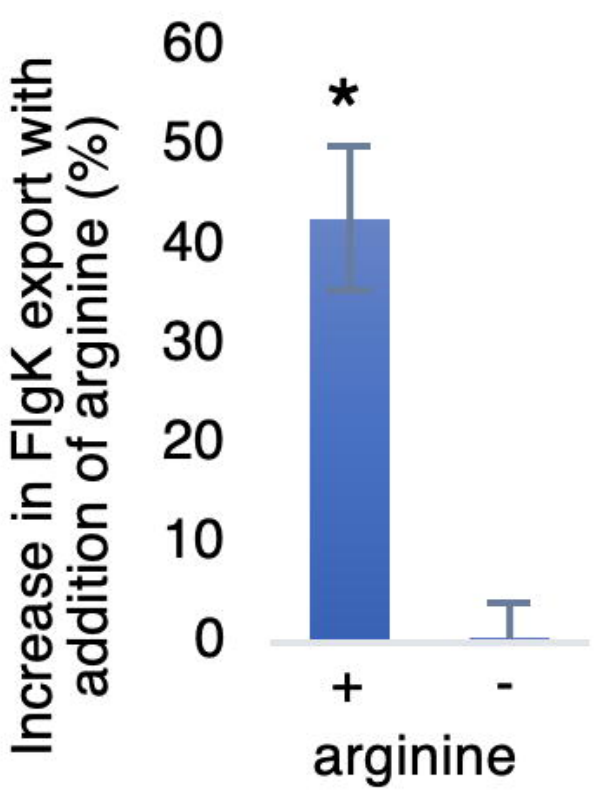

Figure 3 
a

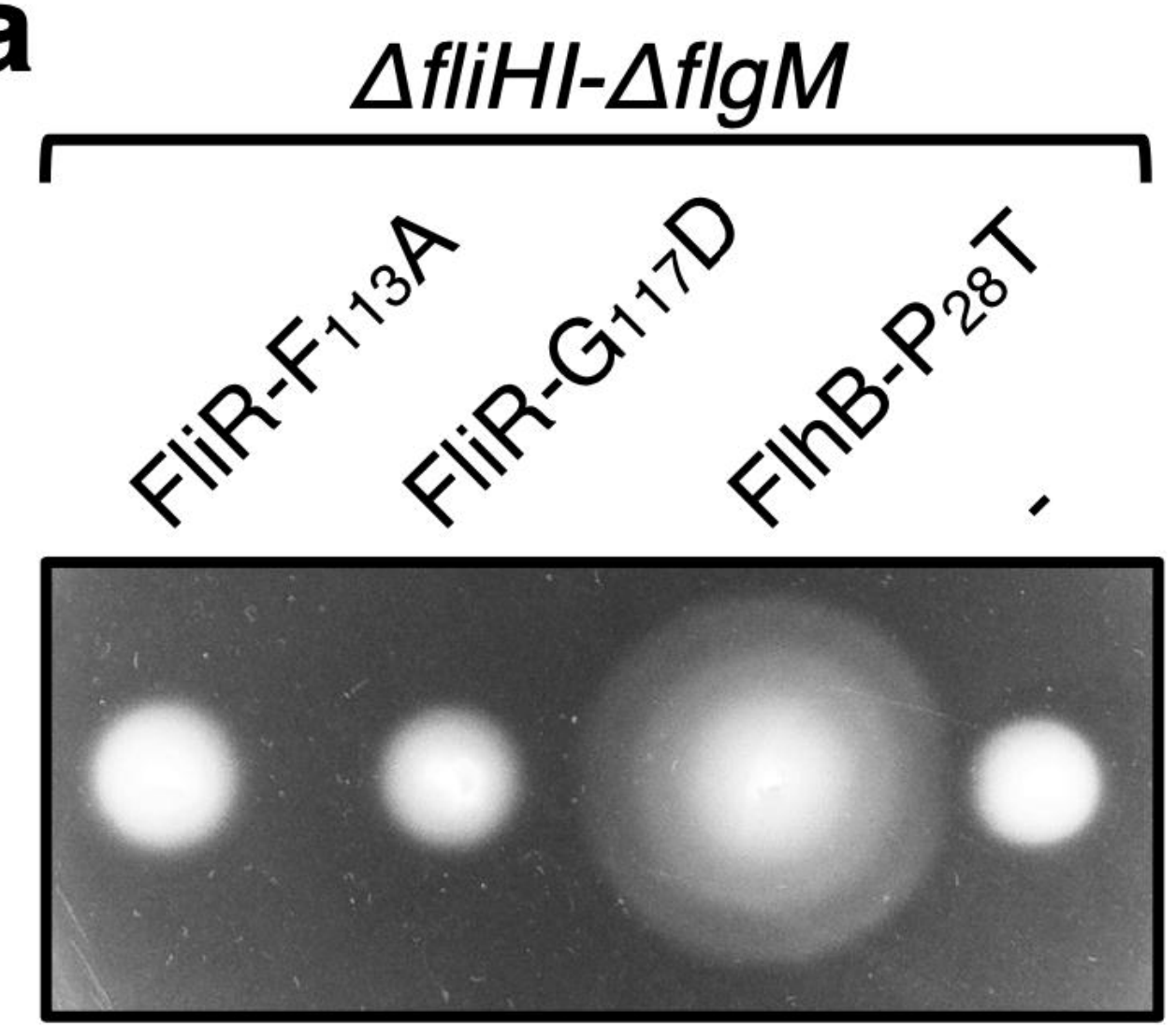

C

FlhB

$\mathrm{P}_{28} \mathrm{~T} \quad \mathrm{FlhB}$

FlgDshort

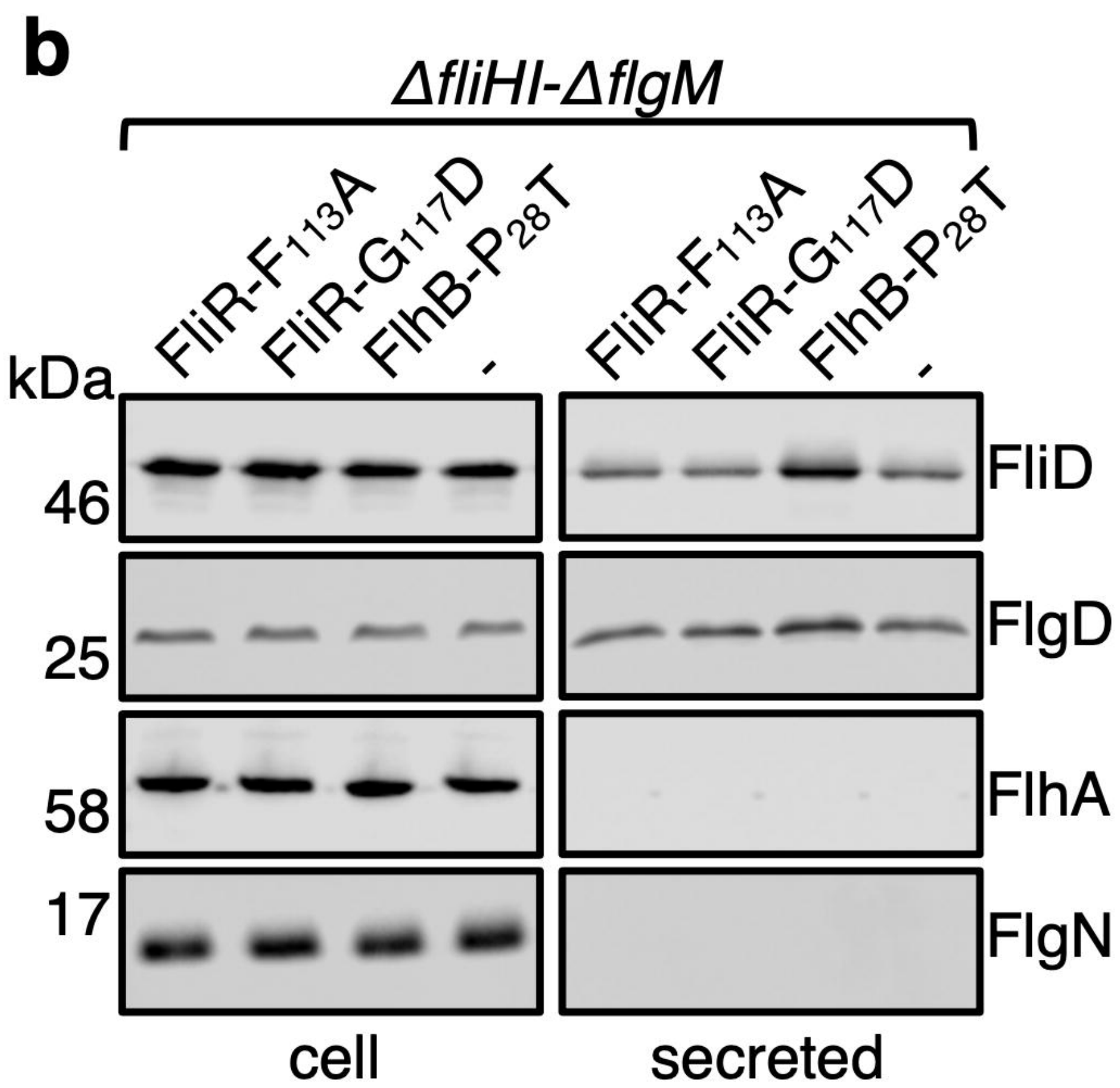

Figure 4 

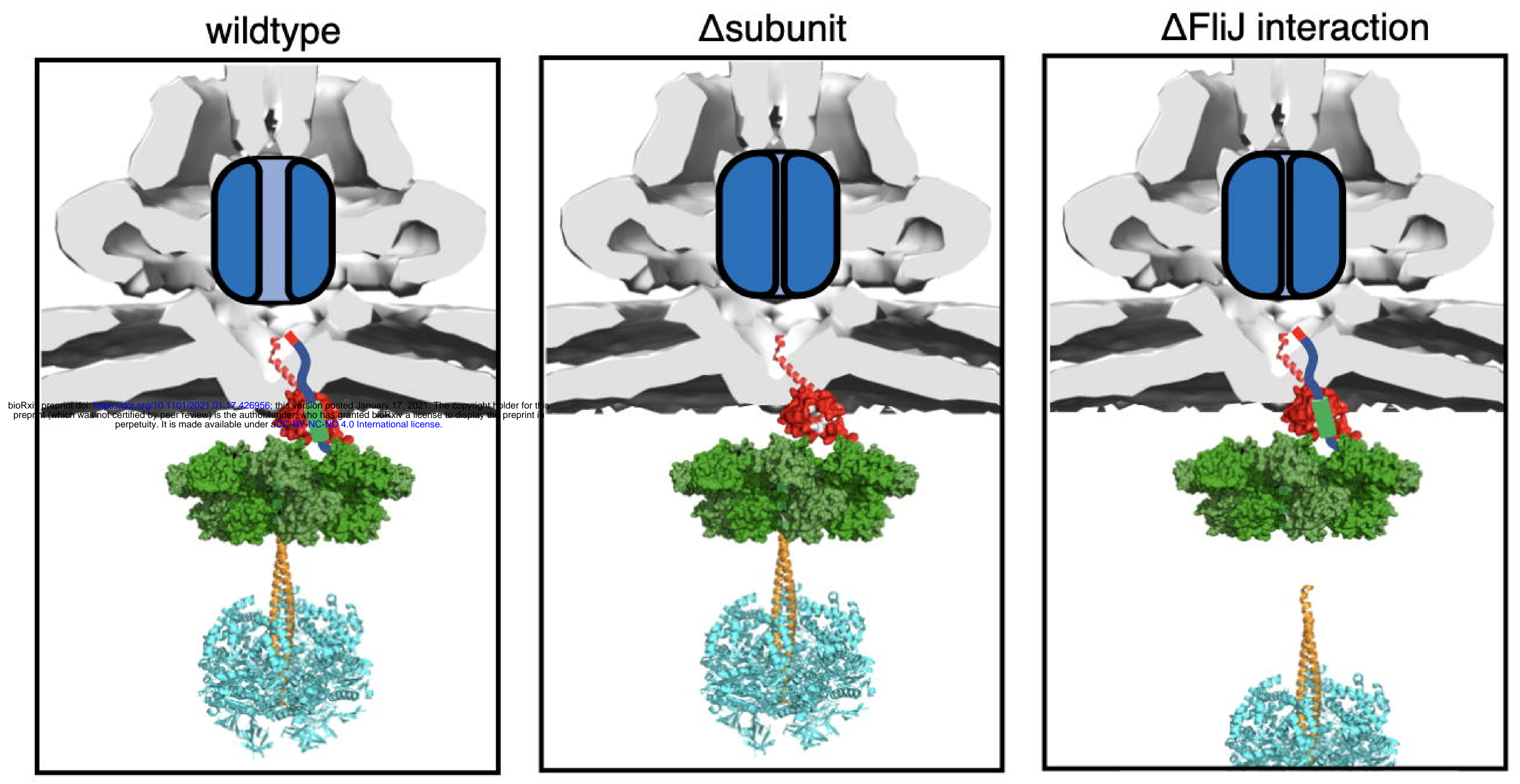

Figure 5 